\title{
Antibacterial Activity of Extract and an Isolated Steroidal Alkaloid from the Seeds of Combretum quadrangulare
} Kurz

\author{
Khesorn Nantachit ${ }^{1}$, Patoomratana Tuchinda ${ }^{2}$, Banyoung Khantawa ${ }^{3}$ and Somjing Roongjang ${ }^{1}$ \\ 1. Department of Pharmaceutical Science, Faculty of Pharmacy, Chiang Mai University 50200, Thailand \\ 2. Department of Chemistry, Faculty of Science, Mahidol University, Paya Thai 10400, Thailand \\ 3. Central Laboratory, Maharaj Nakorn Chiang Mai Hospital, Faculty of Medicine, Chiang Mai Universtiy 50200, Thailand
}

\begin{abstract}
The purpose of this study was to isolate a new compound from the seeds of Combretum quadrangulare Kurz, which showed antibacterial activity. The seeds were macerated with $95 \%$ of ethanol and then purified by column chromatography using Avicel as the adsorbent. After that, $50 \%$ of methanol in water was used as the eluting solvent and continued to further purify by Avicel preparative layer chromatogram 2 times using $80 \%$ of methanol in water as developing solvent. MIC of pure compound was done by two fold dilution method. Muller Hinton agar was selected as the media. The new compound was found as combretin, a steroidal alkaloid, and showed antibacterial activity against Escherichia coli ATCC 25922 and Pseudomonas aeruginosa ATCC 27853 better than Staphylococcus aureus ATCC 25923.
\end{abstract}

Key words: Combretin, steroidal alkaloid, antibacterial activity, Combretum quadrangulare.

\section{Introduction}

Combretum quadrangulare is typically the common plant in Thailand particularly in open wet places. Various therapeutic uses of it were anthelmintics (seeds, roots and leaves) and curing veneral disease (roots and seeds) [1].

For example, alcoholic and other extracts obtained from the roots and seeds could kill earthworms [2]. Also, crude extracts from the seeds showed antibacterial activity. The identified flavonoid compound is combretol. In addition, three compounds of pentacyclic triterpene carboxylic acid, viz $3 \beta, 6 \beta$, 18ß,-trihydroxy-urs-12-en-30-ic acid, 3,6-diketo olean-12-en-28-oi cacid and olean-12-en-28-oic acid were found as the new compounds from roots and seeds and two compounds of long-chain alcohol and amino compound, $\beta$-sitosterol and $\beta$-sito-steryl.

Corresponding author: Khesorn Nantachit, M.S. in Pharm, Department of Pharmaceutical Science, Faculty of Pharmacy, Chiang Mai University, 50200, Thailand, Medicinal chemistry, E-mail address: khesornn@gmail.com.
Other three flavonoids from the flowers are demonstrated i.e. 5-hydroxy-3,7-dimethoxy-2 (3', 4',5', -trimethoxyphenyl)-4H-1benzopyran-4-one(combretol ),5-hydroxy-2-(3'-hydroxy-4'-methoxy-phenyl)-3,7 -dimethoxy-4H-1-benzopyran-4-one (ayanin) and polymorphic form of 5-hydroxy-2-(4'-hydroxy-3',5' -dimethoxyphenyl)-3-7-dimethoxy-4H-1-benzopyran4-one [3].

The seeds of $C$. quadrangulare were revealed anthelmintic activity of roundworms in young buffalo [4]. They found that the number of eggs of Neoascaris vitulorum in feces was reduced after young buffalo ate the seeds and completely disappeared within 1-3 weeks. No toxicity of seed extracts was also observed in albino rat and mice within 2 days.

There are 2 flavonoids, viz, kumatakenin and iso-kaemferide and 3 types of cycloartane triterpenes which were $1 \alpha, 3 \beta$-dihydroxy-cycloart-24-ene-30 -carboxylic acid $1 \alpha, 3 \beta$-dihydroxy-cycloart-24-ene -30-carbo-xylic acid methyl ester and $1 \alpha, 3 \beta-25$ 
-trihydroxy-cycloart-21-al-23-ene-30-carboxylic acid methyl ester from the leaves [5].

The methanol, methanol-water (1:1) and water extracts of the seeds of C. quadrangulare Kurz included triterpene glycosides which demonstrated a good hepatoprotective activity [6]. Similarly, new gallic acid from methanolic extracts of the seeds showed strong hepatoprotective activity [7].

From our preliminary finding, we found that crude methanolic extracts from the seeds of $C$. quadrangulare showed MIC at $312 \mu \mathrm{g} / \mathrm{ml}$ against Staphylococcus aureus ATCC 25923 and demonstrated antibacterial activity against Escherichia coli ATCC 25922, Pseudomonas aeruginosa ATCC 27853 and Klebciella pneumonia ESBL non-producing strain as well [8]. Hence, the objective of this investigation is to isolate new compound that showed antibacterial activity.

\section{Materials and Methods}

This study was conducted as an experimental study to isolate a new compound from the seeds of Combretum quadrangulare for MIC determination.

\subsection{Sample Preparations}

Combretum quadrangulare specimens were collected from Chiang Mai province in 2007. Mature seeds were collected (Nantachit, voucher No.2) and kept in CMU's pharmacy herbarium. The seeds were dried at $40^{\circ} \mathrm{C}$ and powdered. One kilogram of powder was macerated with 3 liters of $95 \%$ of ethanol. Each replicated was macerated for 1 day, filtered and repeated 2 times. The filtrate was evaporated in a vacuum. The residue (crude extract) was brownish black. The percent yield of material collected was 14.38 .

\subsection{Equipment}

NMR (nuclear magnetic resonance) spectra were recorded on a Bruker FT NMR $500 \mathrm{MHz}$ spectrometer. Mass spectrum was recorded by a micrOTOF mass spectrometer.

\subsection{Chemical Reagents}

2.3.1 Column Chormatography Adsorbent: microcrystalline cellulose powder Merck 70-230 mesh (Avicel)

2.3.2 Preparative Chromatography Adsorbent: microcrystalline cellulose powder Merck (Avicel)

2.4 Screening of Crude 95\% of Ethanolic Extract of C. quadrangulare Kurz from 2007 and 2010 by Agar Dilution Method

This screening method was performed as previously described $[9,10]$. Briefly, crude ethanolic extracts of 2007 and 2010 (using sample that was collected in 2007 but kept as dried seeds for 4 years in order to confirm the result of 2007 sample since anti-inflammation action is the objective of the further test) were screened for antibacterial activity against gram positive and gram negative of 3 types of ATCC strains organisms (Table 1). Water was selected to dissolve crude $95 \%$ of ethanolic extract and then two-fold dilution was done by using water as the solvent and mixed with Muller-Hilton agar.

\subsection{Purification of Crude 95\% of Ethanolic Extract in 2010}

$1.5 \mathrm{~g}$ of crude $95 \%$ of ethanolic extract was purified by column chromatography. Avicel was used as the adsorbent. The column was eluted with $50 \%$ of methanol in water. A $10 \mathrm{ml}$ each from total of 3 fractions was collected. Each fraction was sound to produce the same spot in thin layer chromatogram. Each fraction was vacuumed by vacuum pump in order to remove methanol in a cool condition for the reason that the sample fractions might be heat-labile. The remaining water residue was removed by freeze-drying. The residues combined and further purified with preparative thin layer chromatogram (PTLC) twice. Avicel was used as an adsorbent with $1 \mathrm{~mm}$ thickness and developed with $80 \%$ of methanol in water. The pure compound was obtained from second PTLC. 


\subsection{Determining Control Solvent for Antibacterial Test}

Dimethyl sulfoxide, PEG 200, methanol, ethanol, ethyl acetate, dichloromethane and hexane were selected to test for their antibacterial activity by agar diffusion method against 3 pathogenic bacteria ( $S$. aureus, E. coli, P. aeruginosa, ATCC strains). The solvents that showed no antibacterial activity were ethyl acetate and hexane.

2.7 Physical and Spectroscopic Properties of Pure Compound

\subsubsection{Physical Data}

The pure compound was pale yellow solid. Molecular formula of the pure compound is $\mathrm{C}_{52} \mathrm{H}_{83} \mathrm{ON}$ and molecular weight is 737.4804 . The pure compound was soluble in water, methanol and ethylacetate. Melting point could not be determined because the percentage of yield was very low.

\subsubsection{Spectroscopic Data}

\subsubsection{1 ${ }^{1} \mathrm{H}$ NMR Spectrum}

Structure of isolated pure compound based on ${ }^{1} \mathrm{H}$ NMR data was detailed as followed. The first part, 52 protons (Steroidal part), of 33 protons, 15 protons of side chain $\left(\mathrm{C}_{23}-\mathrm{C}_{29}\right) ", \delta=4.9 \mathrm{ppm}, \mathrm{J}=1455.38 \mathrm{~Hz}$ coupling with 3 protons; 2 protons of methylene groups at $\mathrm{C}_{2}$ " position and 1 proton of methane group at $\mathrm{C}_{1}$ " position, $\delta=4.5 \mathrm{ppm}, \mathrm{J}=1369.73 \mathrm{~Hz}$ and coupling with 1 proton of amino group in aziridine ring, $\delta=5.3$ ppm, $\mathrm{J}=1,600 \mathrm{~Hz}$ (Fig. 1 and 3).

The second part, 31 protons (Steroidal skeleton), 20 protons of methylene groups, $\delta=1.3 \mathrm{ppm}, \mathrm{J}=380.92$ $\mathrm{Hz}$ coupling with 2 protons of methylene groups of $\mathrm{C}_{19^{\prime}}$ position, $\delta=2.0 \mathrm{ppm}, \mathrm{J}=612.48 \mathrm{~Hz}$ and coupling with 1 proton of olefinic carbon $\mathrm{C}_{4}{ }^{\prime}$ position, $\delta=2.3 \mathrm{ppm}, \mathrm{J}$ $=694.82 \mathrm{~Hz}$. And, these 20 protons of methylene groups also coupling with 8 protons of angular methine groups, $\delta=0.8 \mathrm{ppm}, \mathrm{J}=264.70 \mathrm{~Hz}$ and $257.40 \mathrm{~Hz}(4$ protons were coupling each other) (Figs. 2 and 3).

\subsubsection{Mass Spectrum}

There were four fragment ions that referred to pure compound. Fragment ion $\mathrm{I}, \mathrm{m} / \mathrm{z}=457.3318$, Fragment ion II, $\mathrm{m} / \mathrm{z}=301$, Fragment ion III, $\mathrm{m} / \mathrm{z}=369.2695$ and Fragment ion IV, $\mathrm{m} / \mathrm{z}=341.2439$ (Fig. 4).

Stereochemistry of ether group was at $\beta$-position and aziridine was at $\alpha$-position (Fig. 5) which were the results of substituted group at 3', 4' position of ring A' (similar to hormone androgen e.g. 4dihydrotestosterone usually was double bond and hydroxyl group). Aziridine ring substituted at 3-position was low stability so the steric hindered at 3-position was easily occurred so fragment ion I was happened (Fig. 4).

Stereochemistry of first part of the pure compound, A-B, B-C, C-D and D-E ring junction should be all trans-configuration in order to form more stability due to large molecules of the first and second part of the

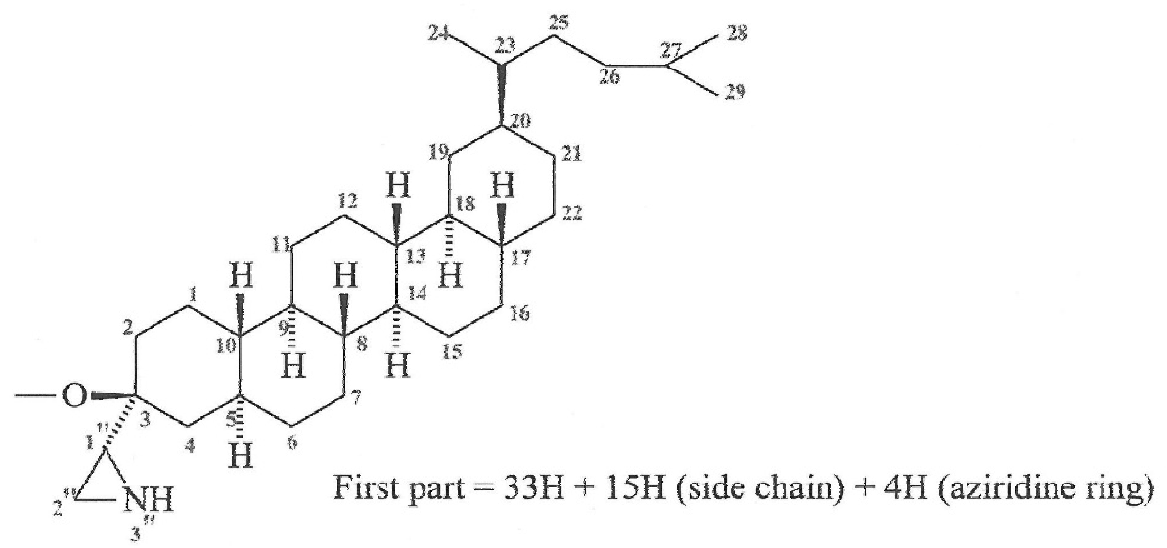

Fig. 1 Structure of the first part of pure compound. 


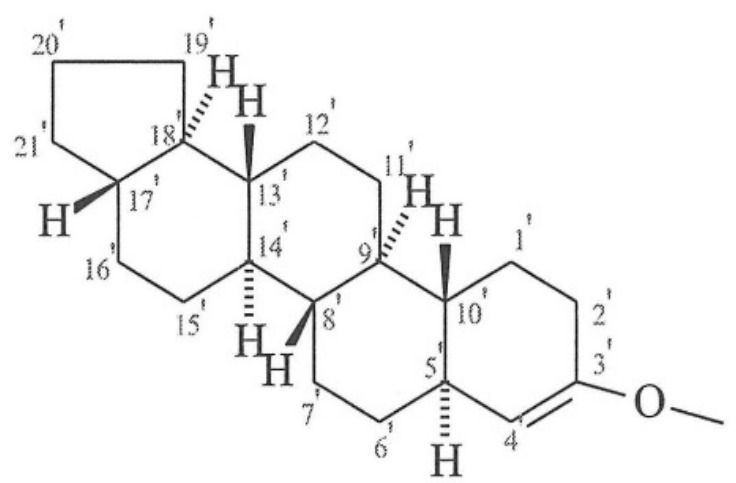

Second part $=31 \mathrm{H}$

Fig. 2 Structure of the second part of pure compound. pure compound which steric hindrance might be occurred easily (Fig. 3). As mentioned above, the pentacyclic triterpene carboxylic acid, $\beta$-sitosterol and $\beta$-sitosteryl were isolated. It showed that chemical constituents of $C$. quadrangulare consisted of terpene and sterol compounds but there was no terpene skeleton at position 3 and 3'. This data confirms that pure compound is a steroroidal compound.

\subsection{Determining MIC (Minimum Inhibitory} Concentration) of the Pure Compound

The method was accomplished by using the similar

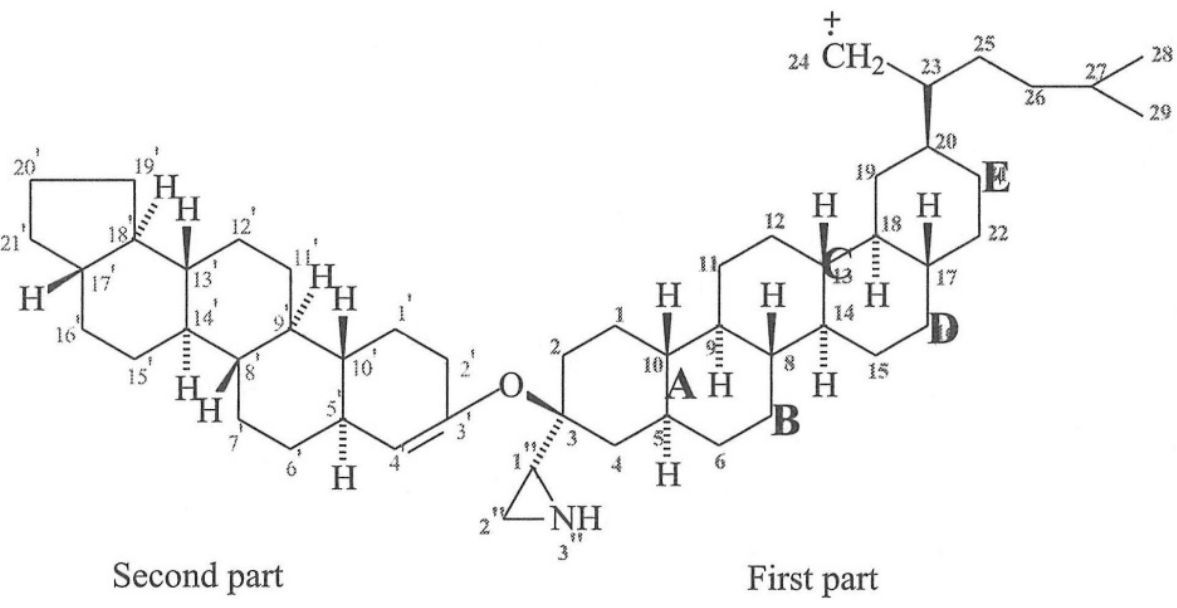

Fig. 3 Molecular ion of pure compound.

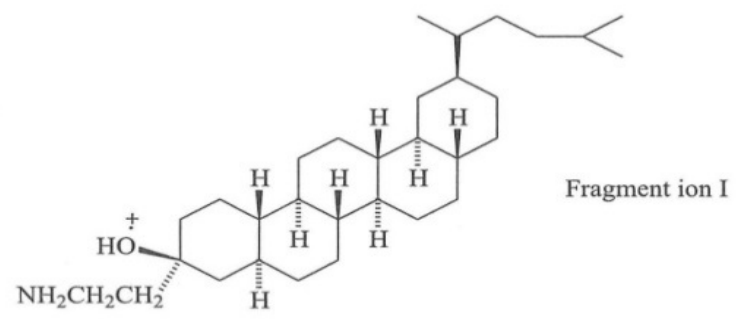

Fragment ion $\mathrm{I}, \mathrm{m} / \mathrm{z}=457.3318$

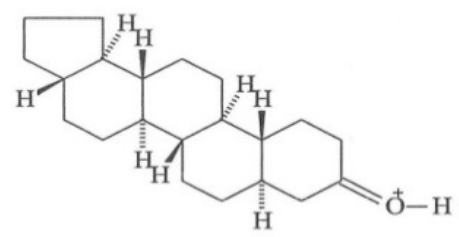

Fragment ion II, $\mathrm{m} / \mathrm{z}=301$

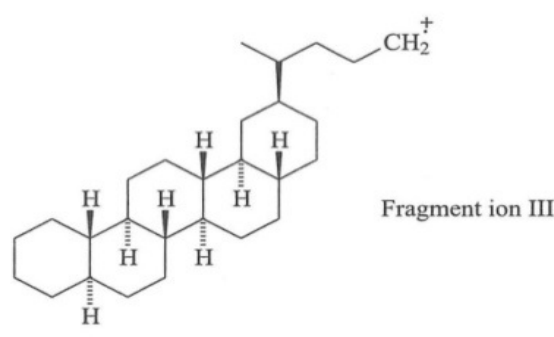

Fragment ion III, $\mathrm{m} / \mathrm{z}=369.2695$

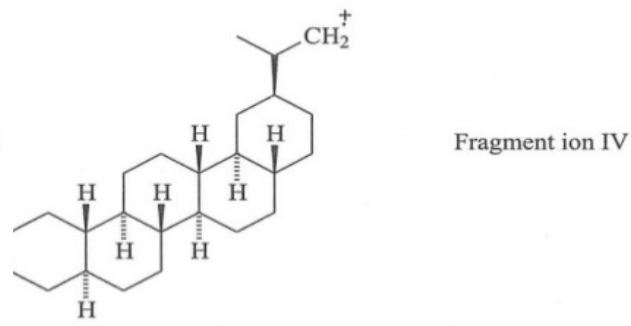

Fragment ion IV, $\mathrm{m} / \mathrm{z}=341.2439$

Fig. 4 Fragment ions I,II, III and IV. 


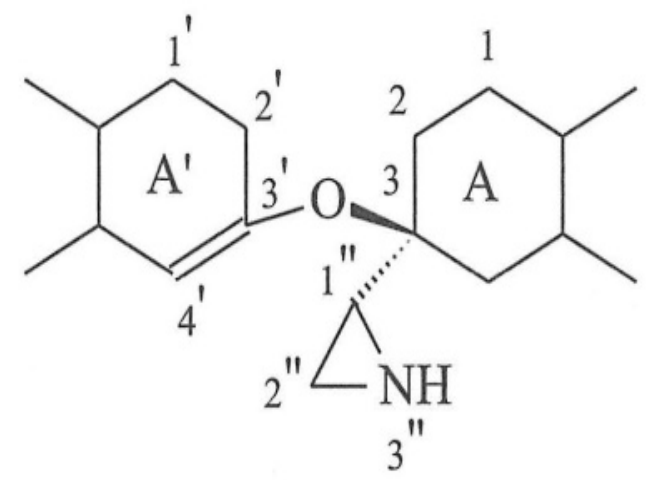

Fig. 5 Stereochemistry of ether group and aziridine ring. procedure as the screening method. Ethyl acetate was used as the solvent due to miscible property with media (Tables 1 and 2).

\section{Results and Discussion}

Three pathogenic bacteria were selected to investigate by agar diffusion method (tested for clear zone). Ethyl acetate was designated as the solvent because of miscible property with media and dissolution of the pure compound.

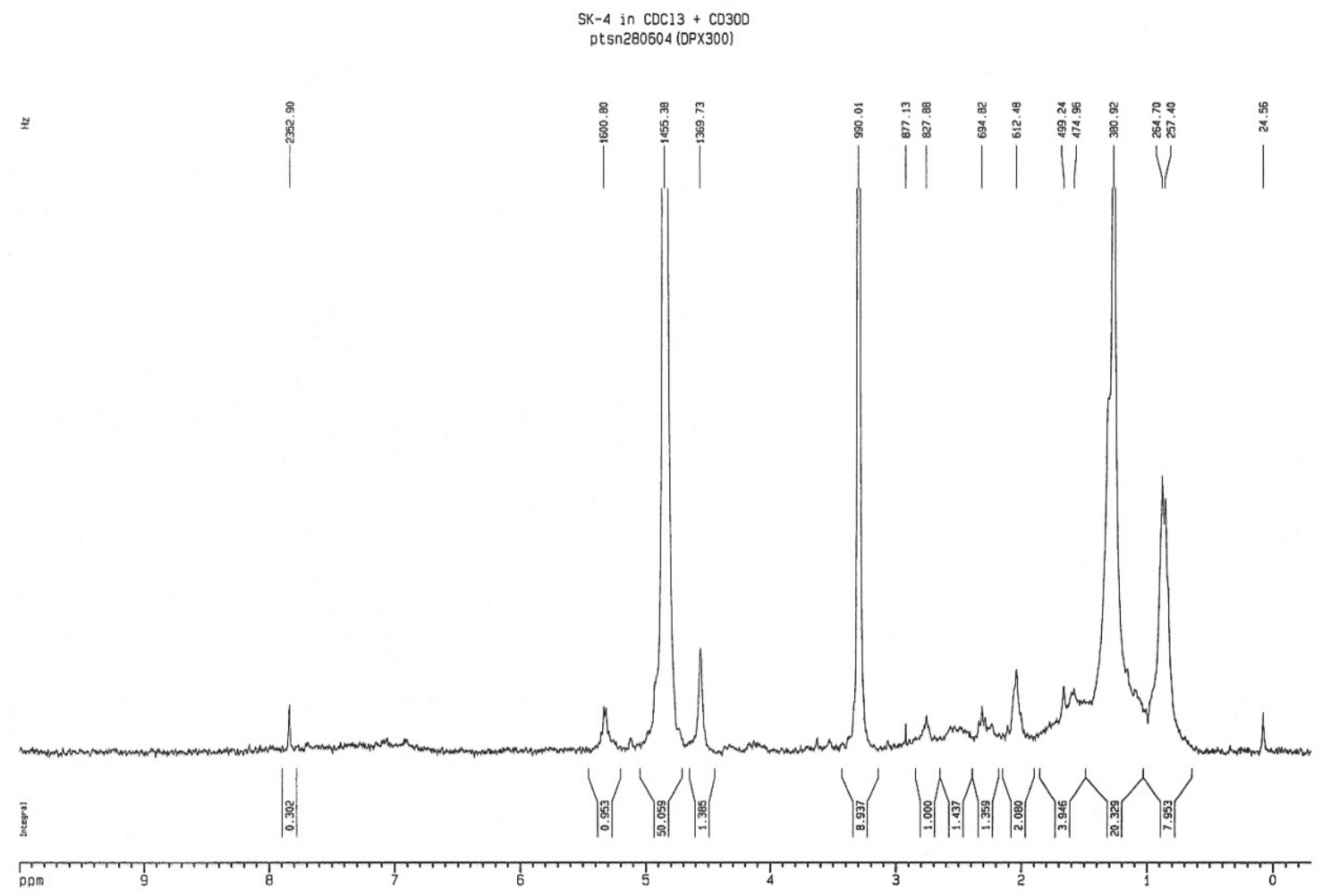

Fig. $6{ }^{1}$ H NMR spectrum of pure compound.

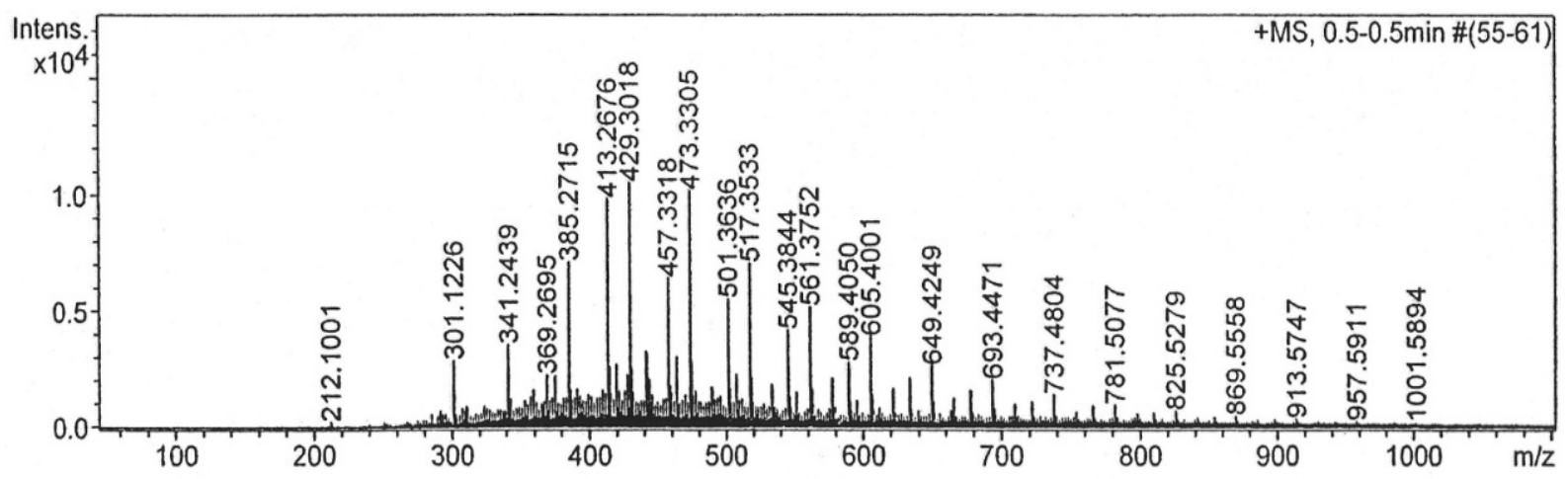

Fig. 7 Mass spectrum of pure compound. 
Table 1 MICs of crude extracts and clear zone of pure compound.

\begin{tabular}{|c|c|c|c|}
\hline No. & Organisms & $\begin{array}{l}\text { MIC I } \\
(\mu \mathrm{g} / \mathrm{ml})\end{array}$ & $\begin{array}{l}\text { MIC II } \\
(\mu \mathrm{g} / \mathrm{ml})\end{array}$ \\
\hline \multicolumn{4}{|c|}{ Crude $95 \%$ of ethanolic extract collected in 2006 and 2007} \\
\hline 1 & A. baumannii $*$ & 937.50 & \\
\hline 2 & S. aureus ATCC 25923 & $\leq 468.75$ & $\leq 468.75$ \\
\hline 3 & E. coli ATCC 25922 & $\overline{15}, 000$ & $\overline{15}, 000$ \\
\hline 4 & P. aeruginosa ATCC 27853 & $1,875.00$ & $1,875.00$ \\
\hline \multicolumn{4}{|c|}{ Crude $95 \%$ of ethanolic extract collect in 2010 (using raw material that collected in 2007 but kept dried seeds for 4 years) } \\
\hline 5 & S. aureus ATCC 25923 & 1,250 & 1,250 \\
\hline 6 & E. coli ATCC 25922 & 5,000 & 5,000 \\
\hline 7 & P. aeruginosa ATCC 27853 & 1,250 & 625 \\
\hline \multicolumn{4}{|c|}{ Screening of pure compound (isolated from crude extract 2010) by agar diffusion method } \\
\hline 8 & Control (Ethyl acetate) & No zone & - \\
\hline 9 & S. aureus ATCC 25923 & No zone & - \\
\hline 10 & E. coli ATCC 25922 & 12 & 12 \\
\hline 11 & P. aeruginosa ATCC 27853 & 11 & 12 \\
\hline
\end{tabular}

Note * was MIC of A. baumannii was done in 2006.

Table 2 MICs of pure compound (isolated from the crude extract 2010) by agar dilution method.

\begin{tabular}{lllll}
\hline No. & $\begin{array}{l}\text { Concentrations } \\
(\boldsymbol{\mu g} / \mathbf{m l})\end{array}$ & $\begin{array}{l}\text { S. aureus } \\
\text { ATCC } 25923\end{array}$ & $\begin{array}{l}\text { E. coli } \\
\text { ATCC 25922 }\end{array}$ & P. aeruginosa ATCC 27853 \\
\hline 1 & 82 & Growth & No growth & No growth \\
2 & 164 & Growth & No growth & No growth \\
3 & 328 & Growth & No growth & No growth \\
4 & 565 & Growth & No growth & No growth \\
5 & 1,312 & Growth & No growth & No growth \\
6 & 2,625 & Growth & Growth* & No growth \\
7 & 5,250 & Growth & Growth & No growth \\
8 & 10,500 & Growth & Growth & Growth** \\
9 & 21,000 & Growth & Growth & Growth \\
10 & Control & No growth & No growth & No growth \\
\hline
\end{tabular}

Note * MIC of E. coli was $2,625 \mu \mathrm{g} / \mathrm{ml}$.

** MIC of P. aeruginosa was $10,500 \mu \mathrm{g} / \mathrm{ml}$.

From our previous study, the crude methanolic extract of C. quadrangulare showed antibacterial activity against gram positive better than gram negative and effective against Acenobacter baumannii (the resistant strain in the hospital) showed MIC at 937.50 $\mu \mathrm{g} / \mathrm{ml}$ (Table 1, No. 1) (unpublished data, [8]). Surprisingly, the seeds were preserved dry over time for 4 years, the isolated pure compound showed antibacterial activity against gram negative better than gram positive (Table 1, No. 2 - No. 7).

From Table 1 and 2, our data demonstrated a similar trend of antibacterial activity. The pure compound exhibited effect against E. coli ATCC strain 25922 (mean of inhibition zone; $12 \mathrm{~mm}$-Table 1, MIC; 2,625 $\mu \mathrm{g} / \mathrm{ml}$-Table 2) which was better than the effect against P. aeruginosa ATCC strain 27853 (mean of inhibition zone; $11.5 \mathrm{~mm}$-Table 1, MIC; 10,500 $\mu \mathrm{g} / \mathrm{ml}$-Table 2). Unfortunately, the effect against S. aureus ATCC strain 25923 could not observed in this study.

From MIC testing method of pure compound, ethyl acetate showed antibacterial action (control test showed no growth of bacteria) (Table 2). This may result from the used solvent was higher amount than that used in agar diffusion method. MIC of E. coli ATCC 25922 was around $2,625 \mu \mathrm{g} / \mathrm{ml}$ and MIC of $P$. aeruginosa ATCC 27853 was about 10,500 $\mu \mathrm{g} / \mathrm{ml}$. In contrast, antibacterial action against E. coli ATCC 25922 and P. aeruginosa ATCC 27853 was found from agar diffusion of pure compound. For the reason that ethyl acetate showed no antibacterial action (Table 1), MIC of E. coli ATCC 25922 and P. aeruginosa ATCC 27853 were subsequently lower than 2,625 and 10,500 


\section{Seeds of Combretum quadrangulare Kurz}

$\mu \mathrm{g} / \mathrm{ml}$ (Table 2).

To overcome the limitation of solvent effect, the control plate may be accomplished first. The used solvent should be $50 \%$ of ethyl acetate in water due to reducing amount of ethyl acetate. The water could sparingly dissolve the pure compound as well.

\section{Conclusion}

From ${ }^{1} \mathrm{H}$ NMR and Mass spectra, the pure compound was steroidal alkaloid named combretin. Combretin showed antibacterial activity against $E$. coli ATCC 25922 and P. aeruginosa ATCC 27853 better than S. aureus ATCC 25923 after dried seeds were kept for 4 years.

\section{Acknowledgement}

The author was grateful to Chiang Mai University and Prof. Dr. Stang Mongkolsuk who was the first investigator on C. quadrangulare in Thailand.

\section{References}

[1] Sachium, P. 1979. Mai Tate Muang Thai. Bangkok. (in Thai)

[2] Aaeman, S., Sukit, W., and Chanpen, V. 1980. "Chemical constituents of C. quadrangulare Kurz." In Proceedings of the $4^{\text {th }}$ Herbs and Spices of Asia.

[3] Ian, R. C., Sydney, R. H., Surachai, N., Somkiat, T., and Allan, H. W. 1985. "The Flavonoid of Combretum quadrangulare. Crystal Structure of the Polymorphic Forms

5-hydroxy-2(4'-hydroxy-3',5'-dimethoxy-phenyl-3,7-dime thoxy-4H-1-benzopyran-4-one." Aust. J. Chem. 38: 117-8.

[4] Perapol, Y., Somporn, S., and Pranee, Y. 1988. "Anthelmintic Activity of the Seeds of C. quadrangulare." In Proceedings of the 15th Veterinarian.

[5] Markus, G., Ernst, P., Ellmerer, M., and Herman, S. 1988. "Cycloartane Triterpenes from Combretum quadrangulare." Phytochemistry. 49 (3): 835-8.

[6] Arjan, H. B., Tezuka, Y. I., Ketut, A., Xiang, Q., Hase, K., Tran, K. Q., et al. 2000. "Hepatoprotective Constituents of the Seeds of Combretum quadrangulare Kurz." Biol. Pharm. Bull. 23 (4): 456-60.

[7] Arjan, H. B., Yasuhiro, T., Kim, Q. T., Ken, T., Ikus, S., and Shigetoshi, K. 2000. "Methyl Quadrangulare A-D and Related Triterpenes from Combretum quadrangulare." Chem. Pharm. Bull. (Tokyo). 48 (4): 496-504.

[8] Khesorn, N., Dumrong, S., and Banyong, K. 2006. "Antibacterial Activity of the Seeds of Combretum quadrangulare Kurz (combretaceae).” CMU. Journal. 5 (3): 333-9.

[9] John, A. W. 1981. Laboratory Procedure in Clinical Microbiology. New York: Springer-Verlag.

[10] Edwin, H. I. 1988. Manual of Clinical Microbiology. 3rd ed. Washington DC. American Society for Microbiology. 